TAIWANESE JOURNAL OF MATHEMATICS

Vol. 12, No. 7, pp. 1805-1825, October 2008

This paper is available online at http://www.tjm.nsysu.edu.tw/

\title{
A SCHUR-NEWTON ALGORITHM FOR ROBUST POLE ASSIGNMENT OF DESCRIPTOR SYSTEMS
}

\author{
Tiexiang Li and Eric King-wah Chu
}

\begin{abstract}
We propose an algorithm for the pole assignment problem for descriptor systems with proportional and derivative state feedback. The algorithm is the first of its kind, making use of the Schur form and minimizing the departure from normality of the closed-loop poles by Newton's method. Three illustrative examples are given.
\end{abstract}

\section{INTRODUCTION}

We consider the robust pole assignment of the descriptor system (RPAP_DS)

$$
E \lambda x(t)=A x(t)+B u(t)
$$

where $x(t) \in \mathbb{R}^{n}, u(t) \in \mathbb{R}^{m}, A, B$ and $E$ are real matrices of appropriate dimensions with $E$ possibly singular and $\lambda x(t)=\dot{x}(t)$ (or $x(t+1)$ ) for a continuous- (or discrete-) time system. We assume that the pencil $(A, E)$ is regular or $\operatorname{det}(\alpha A-\beta E) \not \equiv 0$, and that the system $(A, E, B)$ is controllable [24], i.e. $\operatorname{rank}(\alpha A-\beta E, B)=n=$ $\operatorname{rank}(E, B)$, for arbitrary $\alpha, \beta \in \mathbb{C}$. Also consult $[2,4,11]$ on the issue of controllability and the related problem of regularization.

By robust pole assignment (RPAP), we mean to seek feedback matrices $F$ and $G$ so that the closed-loop pencil $(A+B F, E+B G)$ possesses a prescribed desirable spectrum. It is equivalent to modifying the system in (1) with proportional and derivative state feedback $u=F x-G \dot{x}$. It is well-known [24] that our problem is solvable when the control system (1) is controllable.

There have been many previous attempts in tackling RPAPs. For ordinary systems, please consult $[3,6,8,16,17,21]$ and the references therein. Second-order systems have been considered in $[7,9]$ and descriptor systems in $[2,5,10,23,24]$ (some with

Received January 8, 2007, accepted June 11, 2007.

Communicated by Wen-Wei Lin.

2000 Mathematics Subject Classification: 15A22, 93B52, 93B55.

Key words and phrases: Descriptor systems, Robust pole assignment, Schur form. 
only proportional feedback). Robustness is optimized directly in [6-10, 15-17, 23, 24] and indirectly in $[3,5]$. For ordinary systems, a method in [16] has been implemented in the MATLAB command place [18]. The Schur form [12] has been utilized in pole assignment problems in $[3,5,8,17,21,22]$. However, robustness is not directly optimized in $[3,5]$ and the Schur form is not directly computed in [21, 22]. A Schur form optimizing the robustness measure of the departure from normality for ordinary systems is computed directly in [8] in a non-iterative manner. Only suboptimality is achieved in [8], due to the freedom in the first Schur vector, but full optimality is possible after a Newton refinement step [17].

In this paper, our methods for descriptor systems is a generalization of the ones in $[8,17]$ for ordinary systems. This Schur-Newton algorithm represents the only known method which minimizes the departure from normality of the closed-loop system by computing directly the closed-loop Schur form.

Lastly, there are many concepts of controllability for descriptor systems and many different possibilities in measuring robustness. Various optimization techniques can be applied to these robustness measures, as in [23, 24]. Comparing methods under different assumptions or optimizing different robustness measures is difficult, if at all possible. Optimizing robustness measures blindly usually runs into slow convergence, the lack of a good feasible starting value or other related problems. The main contribution of this paper is the availability of a good feasible starting value, from the Schur algorithm in Section 3, which can be refined efficiently by the Schur-Newton refinement in Section 4. The numerical examples in Section 5 show much promise for the SchurNewton algorithm but more thorough testing will have to be done.

\section{Departure From Normality}

We shall quote the departure from normality measure for generalized eigenvalue problems [19], generalizing the similar measure for ordinary systems $[1,12,14,20]$.

Definition 2.1. Let $\{A, B\}$ be a regular matrix pair and $\mathcal{U}_{\{A, B\}}$ be the set of all pairs of transformations $\{Z, U\}$ which satisfy the following conditions:

(i) $Z, U \in \mathbb{C}^{n \times n}, Z$ is nonsingular and $U$ is unitary;

(ii) $Z^{-1} A U$ and $Z^{-1} B U$ are both upper triangular; and

(iii) $\left|\left(Z^{-1} A U\right)_{i i}\right|^{2}+\left|\left(Z^{-1} B U\right)_{i i}\right|^{2}=1(i=1, \cdots, n)$ where $(A)_{i j}$ is the $(i, j)$ element of $A$.

Let $(Z, U) \in \mathcal{U}_{\{\mathcal{A}, \mathcal{B}\}}$ and $\operatorname{diag}(A) \in \mathbb{C}^{n}$ denote the diagonal matrix sharing the diagonal of $A$. Denote

$$
\mu(Z, U) \equiv \|\left(Z^{-1} A U-\operatorname{diag}\left(Z^{-1} A U\right), Z^{-1} B U-\operatorname{diag}\left(Z^{-1} B U\right) \|_{2}\right.
$$


and

$$
\Delta_{2}(A, B) \equiv \inf _{\{Z, U\} \in \mathcal{U}_{\{\mathcal{A}, \mathcal{B}\}}} \mu(Z, U) .
$$

Then $\Delta_{2}(A, B)$ is called the departure from normality measure of $\{A, B\}$.

Definition 2.2. Let $\sigma(A, B)=\left\{\left(\alpha_{i}, \beta_{i}\right)\right\}$ denote the spectrum of the pencil $\alpha A-\beta B$ and let $(\alpha, \beta) \in \sigma(C, D)$. The spectral variation of $(C, D)$ from $(A, B)$ equals

$$
s_{(A, B)}(C, D) \equiv \max _{(\alpha, \beta)}\left\{s_{(\alpha, \beta)}\right\}, \quad s_{(\alpha, \beta)} \equiv \min _{i}\left\{\left|\alpha \beta_{i}-\beta \alpha_{i}\right|\right\}
$$

Theorem 2.1. (Henrici Theorem [19]). Let $\{A, B\}$ and $\{C, D\}$ be regular pairs of the same dimension, $\Delta_{2}(A, B)$ is the departure from normality measure of $\{A, B\}$, and suppose $\Delta_{2}(A, B) \neq 0$. Let $W=(A, B)$ and $\widetilde{W}=(C, D)$, then

$$
s_{(A, B)}(C, D) \leq \frac{\eta}{g(\eta)}\left[1+\Delta_{2}(A, B)\right] d_{2}(W, \widetilde{W})
$$

where $d_{2}(W, \widetilde{W})=\|\sin \Theta(W, \widetilde{W})\|_{2}$ denotes the distance between $W$ and $\widetilde{W}$,

$$
\eta=\frac{\Delta_{2}(A, B)}{\left[1+\Delta_{2}(A, B)\right] d_{2}(W, \widetilde{W})},
$$

and $g(\eta)$ is the unique nonnegative root of $g+g^{2}+\cdots+g^{n}=\eta(\eta>0)$.

Based on Theorem 2.1, we shall minimize the departure from normality of the closed-loop matrix pencil in the effort to control the robustness of the closed-loop spectrum or system.

\section{SCHUR Algorithm}

Multiplying the nonsingular matrix $Z^{-1}$ and orthogonal matrix $X$ on the both sides of $A+B F$ and $E+B G$, we get

$$
Z^{-1}(A+B F) X=D_{\alpha}+N_{\alpha}, \quad Z^{-1}(E+B G) X=D_{\beta}+N_{\beta},
$$

or

$$
(A+B F) X=Z\left(D_{\alpha}+N_{\alpha}\right), \quad(E+B G) X=Z\left(D_{\beta}+N_{\beta}\right),
$$

where $D_{\alpha}, D_{\beta}$ are diagonal, and $N_{\alpha}, N_{\beta}$ are straightly upper triangular. 
Assuming without loss of generality that the feedback matrix $B$ has full rank and possesses the QR decomposition

$$
B=\left[Q_{1}, Q_{2}\right]\left[\begin{array}{c}
R_{B} \\
0
\end{array}\right]=Q_{1} R_{B}
$$

then $Q_{2}^{\top} B=0$ and $B^{\dagger}=R_{B}^{-1} Q_{1}^{\top}$.

Pre-multiplying the equations in (2), respectively, by $Q_{2}^{\top}$ and $B^{\dagger}$, we obtain

$$
Q_{2}^{\top} A X-Q_{2}^{\top} Z D_{\alpha}-Q_{2}^{\top} Z N_{\alpha}=0, \quad Q_{2}^{\top} E X-Q_{2}^{\top} Z D_{\beta}-Q_{2}^{\top} Z N_{\beta}=0,
$$

and

(4) $\quad F=R_{B}^{-1} Q_{1}^{\top}\left[Z\left(D_{\alpha}+N_{\alpha}\right) X^{\top}-A\right], \quad G=R_{B}^{-1} Q_{1}^{\top}\left[Z\left(D_{\beta}+N_{\beta}\right) X^{\top}-E\right]$.

For a given eigenvalue pairs $\left\{D_{\alpha}, D_{\beta}\right\}$, we can select $Z, X$ from (3) then obtain the solution to the pole assignment problem using (4).

In this paper, we denote $C \oplus D=\left[\begin{array}{cc}C & 0 \\ 0 & D\end{array}\right]$ (where $C, D$ need not to be square),

$X=\left[x_{1}, x_{2}, \cdots, x_{n}\right] \in \mathbb{R}_{\mathbb{B}}^{n \times n}, \quad v(X)=\left[x_{1}^{\top}, x_{2}^{\top}, \cdots, x_{n}^{\top}\right]^{\top}, \quad v(A X B)=\left(B^{\top} \otimes\right.$ A) $v(X)$,

$\operatorname{Vec}(I)=[1|0,1| 0,0,1|\cdots| 0, \cdots, 0,1]^{\top} \in \mathbb{R}^{n(n+1) / 2 \equiv q}$. Note that both $v(\cdot)$ and $\operatorname{Vec}(\cdot)$ stack columns of matrices but the latter discards zeroes for strictly upper triangular matrices.

\subsection{Real Eigenvalues}

Let us first consider the case when all the closed-loop eigenvalues are real, with the closed-loop system matrix pair $\left(A_{c}, E_{c}\right)=(A+B F, E+B G)=\left(Z \Lambda_{\alpha} X^{\top}, Z\right.$ $\left.\Lambda_{\beta} X^{\top}\right)$ in Schur form. Here we have $\left(\Lambda_{\alpha}, \Lambda_{\beta}\right)=\left(D_{\alpha}+N_{\alpha}, D_{\beta}+N_{\beta}\right)$, with $D_{\alpha}=\operatorname{diag}\left\{\alpha_{1}, \cdots, \alpha_{n}\right\}, D_{\beta}=\operatorname{diag}\left\{\beta_{1}, \cdots, \beta_{n}\right\}$ being real, $N_{\alpha}=\left[\hat{\eta}_{1}, \hat{\eta}_{2}, \cdots, \hat{\eta}_{n}\right]$, $N_{\beta}=\left[\hat{\zeta}_{1}, \hat{\zeta}_{2}, \cdots, \hat{\zeta}_{n}\right]$ being straightly upper triangular and nilpotent, and $\eta_{j}=$ $\left[\eta_{1, j}, \cdots, \eta_{j-1, j}\right]^{\top}, \zeta_{j}=\left[\zeta_{1, j}, \cdots, \zeta_{j-1, j}\right]^{\top}$ are the vectors constructed from $\hat{\eta}_{j}$ and $\hat{\zeta}_{j}$ with the zeroes at the bottom deleted (thus $\eta_{1}, \zeta_{1}$ are degenerate and $\eta_{j}, \zeta_{j} \in \Re^{j-1}$ ). The Schur vector matrix $X$ is orthogonal. The case when some of the eigenvalues of $\Lambda$ are complex will be considered later. From (3), for $j=1,2, \cdots, n$, we then have

$$
Q_{2}^{\top} A x_{j}-\alpha_{j} Q_{2}^{\top} z_{j}-Q_{2}^{\top} \sum_{k=1}^{j-1} \eta_{k j} z_{k}=0=Q_{2}^{\top} E x_{j}-\beta_{j} Q_{2}^{\top} z_{j}-Q_{2}^{\top} \sum_{k=1}^{j-1} \zeta_{k j} z_{k} .
$$

With $X_{-j} \equiv\left[x_{1}, \cdots, x_{j-1}\right], Z_{-j} \equiv\left[z_{1}, \cdots, z_{j-1}\right](j \geq 2)$, we can select $x_{j}, z_{j}, \eta_{j}$ and $\zeta_{j}$ from

$$
Q_{2}^{\top} A x_{j}-\alpha_{j} Q_{2}^{\top} z_{j}-Q_{2}^{\top} Z_{-j} \eta_{j}=0=Q_{2}^{\top} E x_{j}-\beta_{j} Q_{2}^{\top} z_{j}-Q_{2}^{\top} Z_{-j} \zeta_{j} .
$$


In order to obtain the Schur decomposition $\left(A_{c}, E_{c}\right)=(A+B F, E+B G)=\left(Z \Lambda_{\alpha} X^{\top}\right.$, $\left.Z \Lambda_{\beta} X^{\top}\right)$ with $X$ being unitary and $Z$ being nonsingular, we select $X_{-j}^{\top} x_{j}=0=$ $Z_{-j}^{\top} z_{j}$ and $\left\|x_{j}\right\|=1$. Consequently, we select $x_{j}, z_{j}, \eta_{j}$ and $\zeta_{j}$ from

$$
M_{j}\left[\begin{array}{c}
x_{j} \\
z_{j} \\
\eta_{j} \\
\zeta_{j}
\end{array}\right]=0, \quad M_{j} \equiv\left[\begin{array}{cccc}
Q_{2}^{\top} A & -\alpha_{j} Q_{2}^{\top} & -Q_{2}^{\top} Z_{-j} & 0 \\
Q_{2}^{\top} E & -\beta_{j} Q_{2}^{\top} & 0 & -Q_{2}^{\top} Z_{-j} \\
X_{-j}^{\top} & 0 & 0 & 0 \\
0 & Z_{-j}^{\top} & 0 & 0
\end{array}\right] .
$$

Notice that the null space $\mathcal{N}\left(M_{j}\right)$ above is non-empty and the algorithm is feasible, as its dimension lies between $2 m$ and $2(m+j-1)$.

From Theorem 2.1, we can minimize the size of $\left(\eta_{j}, \zeta_{j}\right)$, leading to the optimal departure from normality measure and robustness of the closed-loop spectrum. From here on, $\left\|N_{\alpha}\right\|^{2}+\left\|N_{\beta}\right\|^{2}$, the departure from normality, is the robustness measure in the RPAP_DS. We thus arrive to the subproblem from which $x_{j}, z_{j}, \eta_{j}$ and $\zeta_{j}$ are chosen: (for $j>1$ )

$$
\min _{\left\|x_{j}\right\|=1}\left\|\eta_{j}\right\|_{F}^{2}+\left\|\zeta_{j}\right\|_{F}^{2} \quad \text { subject to } M_{j}\left[\begin{array}{c}
x_{j} \\
z_{j} \\
\eta_{j} \\
\zeta_{j}
\end{array}\right]=0 .
$$

Let $\left[S_{j 1}^{\top}, S_{j 2}^{\top}, S_{j 3}^{\top}, S_{j 4}^{\top}\right]^{\top}$ be a unitary basis of the null space of $M_{j}$, with $x_{j}=S_{j 1} u_{j}$, $z_{j}=S_{j 2} u_{j}, \eta_{j}=S_{j 3} u_{j}$ and $\zeta_{j}=S_{j 4} u_{j}$. It is easy to see that $x_{j}, z_{j}, \eta_{j}$ and $\zeta_{j}$ can be chosen, for a given value of $j$, by finding the smallest generalized singular value (GSV) [12] and its associated singular vector for $\left\{\left(S_{j 1}^{\top}, S_{j 2}^{\top}\right)^{\top},\left(S_{j 3}^{\top}, S_{j 4}^{\top}\right)^{\top}\right\}$.

For a given ordering of the close-loop poles, $x_{1}, z_{1}$ are constrained in (6) but cannot be chosen uniquely, as $\eta_{1}, \zeta_{1}$ are degenerate. Similar comments apply in the complex case below.

\subsection{Complex Eigenvalues}

When some of the closed-loop eigenvalues are complex, we can modify our algorithm using real arithmetic so that a real feedback matrix $F, G$ can be obtained. Using the following modified real Schur form, the real vectors $x_{j}, x_{j+1}, z_{j}, z_{j+1}, \eta_{j}, \eta_{j+1}$ and $\zeta_{j}, \zeta_{j+1}$ are chosen via

$$
\begin{gathered}
Q_{2}^{\top} A\left[x_{j}, x_{j+1}\right]-Q_{2}^{\top}\left[z_{j}, z_{j+1}\right] D_{\alpha j}-Q_{2}^{\top} Z_{-j}\left[\eta_{j}, \eta_{j+1}\right]=0 \\
Q_{2}^{\top} E\left[x_{j}, x_{j+1}\right]-Q_{2}^{\top}\left[z_{j}, z_{j+1}\right] D_{\beta j}-Q_{2}^{\top} Z_{-j}\left[\zeta_{j}, \zeta_{j+1}\right]=0, \quad X_{-j}^{\top}\left[x_{j}, x_{j+1}\right]=0
\end{gathered}
$$


where

$$
D_{\alpha j}=\left[\begin{array}{rr}
\mu_{j} & \nu_{j} \\
-\nu_{j} & \mu_{j}
\end{array}\right], \quad D_{\beta j}=\left[\begin{array}{rr}
\kappa_{j} & \tau_{j} \\
-\tau_{j} & \kappa_{j}
\end{array}\right] .
$$

Equivalently, we have

$$
\begin{gathered}
\\
M_{j}\left[\begin{array}{c}
x_{j} \\
x_{j+1} \\
z_{j} \\
z_{j+1} \\
\hline \eta_{j} \\
\eta_{j+1} \\
\zeta_{j} \\
\zeta_{j+1}
\end{array}\right]=0, \quad M_{j} \\
\equiv\left[\begin{array}{cccc}
I_{2} \otimes\left(Q_{2}^{\top} A\right) & D_{\alpha j}^{\top} \otimes Q_{2}^{\top} & -I_{2} \otimes Q_{2}^{\top} Z_{-j} & 0 \\
I_{2} \otimes\left(Q_{2}^{\top} E\right) & D_{\beta j}^{\top} \otimes Q_{2}^{\top} & 0 & -I_{2} \otimes Q_{2}^{\top} Z_{-j} \\
I_{2} \otimes X_{-j}^{\top} & 0 & 0 & 0 \\
0 & I_{2} \otimes Z_{-j}^{\top} & 0 & 0
\end{array}\right]
\end{gathered}
$$

while minimizing $\left\|\left[\eta_{j}, \eta_{j+1}\right]\right\|_{F}^{2}+\left\|\left[\zeta_{j}, \zeta_{j+1}\right]\right\|_{F}^{2}$ with $\left\|\left[x_{j}, x_{j+1}\right]\right\|_{F}=1$. Notice that the null space $\mathcal{N}\left(M_{j}\right)$ in (7) is non-empty and the algorithm is feasible, as its dimension lies between $4 m$ and $4(m+j-1)$. Also, we have not imposed the quadratic constraint that $x_{j} \perp x_{j+1}$. The conditioning of the pseudo-Schur vectors $\left[x_{j}, x_{j+1}\right]$ is then controlled by $\left\|\left[\eta_{j}, \eta_{j+1}\right]\right\|_{F}^{2}+\left\|\left[\zeta_{j}, \zeta_{j+1}\right]\right\|_{F}^{2}$, or the size of the upper triangular parts of $\Lambda_{\alpha}, \Lambda_{\beta}$ corresponding to the complex conjugate pair of eigenvalues in $\left(D_{\alpha j}, D_{\beta j}\right)$. Note, in the real Schur form, that $\left(D_{\alpha j}, D_{\beta j}\right)$ can be replaced by any matrix pair with the same eigenvalues, with $x_{j}^{\top} x_{j+1}=0$. This orthogonality condition is a difficult quadratic constraint and is abandoned for simplicity. To pay for this simplicity, the pseudo-Schur vector matrix $X$ is no longer orthogonal. However, it is still nearly orthogonal, with $X^{\top} X$ being block-diagonal and $2 \times 2$ diagonal blocks for individual complex conjugate pairs of closed-loop eigenvalues. The conditioning of the eigenvalues are then partly controlled by the sizes of $\eta_{j}, \eta_{j+1}$ in $N_{\alpha}$ and $\zeta_{j}, \zeta_{j+1}$ in $N_{\beta}$.

Let $\left[S_{j 1}^{\top}, S_{j 2}^{\top}, S_{j 3}^{\top}, S_{j 4}^{\top}, S_{j 5}^{\top}, S_{j 6}^{\top}, S_{j 7}^{\top}, S_{j 8}^{\top}\right]^{\top}$ be a unitary basis for the null space defined in (7). We are looking for the vectors

$$
\begin{aligned}
& x_{j}=S_{j 1} u_{j}, \quad x_{j+1}=S_{j 2} u_{j}, \quad z_{j}=S_{j 3} u_{j}, \quad z_{j+1}=S_{j 4} u_{j}, \\
& \eta_{j}=S_{j 5} u_{j}, \quad \eta_{j+1}=S_{j 6} u_{j}, \quad \zeta_{j}=S_{j 7} u_{j}, \quad \zeta_{j+1}=S_{j 8} u_{j}
\end{aligned}
$$


which satisfy $\min _{u_{j}}\left\|\left[\eta_{j}, \eta_{j+1}\right]\right\|_{F}^{2}+\left\|\left[\zeta_{j}, \zeta_{j+1}\right]\right\|_{F}^{2}$ s.t. $\left\|\left[x_{j}, x_{j+1}\right]\right\|_{F}^{2}=1$, or $\min _{u_{j}} u_{j}^{\top}\left(S_{j 5}^{\top} S_{j 5}+S_{j 6}^{\top} S_{j 6}\right) u_{j}+u_{j}^{\top}\left(S_{j 7}^{\top} S_{j 7}+S_{j 8}^{\top} S_{j 8}\right) u_{j} \quad$ s.t. $u_{j}^{\top}\left(S_{j 1}^{\top} S_{j 1}+S_{j 2}^{\top} S_{j 2}\right) u_{j}=1$

Similar to the real case earlier, the minimization can be achieved via the GSVs of the matrix pair $\left\{\left(S_{j 1}^{\top}, S_{j 2}^{\top}, S_{j 3}^{\top}, S_{j 4}^{\top}\right)^{\top},\left(S_{j 5}^{\top}, S_{j 6}^{\top}, S_{j 7}^{\top}, S_{j 8}^{\top}\right)^{\top}\right\}$.

Remark. In Theorem 2.1, $Z$ is only required to be nonsingular, but this will be difficult to achieve in practice. If it is unconstrained, an ill-conditioned $Z$ may cause problems in the Schur-Newton refinement in the next Section. Consequently, we require in the calulcations in (5) and (7) that $Z$ has orthogonal columns.

\section{Schur-Newton Optimization Algorithm}

From the Schur algorithm in Section 3, we obtain the starting value for the Newton refinement technique in this Section.

\subsection{Real Eigenvalues}

We seek feedback matrices $F, G \in \mathbb{R}^{m \times n}$ such that

$$
\lambda(A+B F, E+B G)=\lambda\left\{\left[\left(\begin{array}{cc}
\Lambda & 0 \\
0 & I
\end{array}\right),\left(\begin{array}{ll}
I & 0 \\
0 & 0
\end{array}\right)\right]\right\}=\lambda\left(D_{\alpha}, D_{\beta}\right)
$$

for given $D_{\alpha} \equiv \operatorname{diag}\left\{\alpha_{1}, \cdots, \alpha_{n}\right\}, D_{\beta} \equiv\left\{\beta_{1}, \cdots, \beta_{n}\right\}$. We have $(A+B F) Y D_{\beta}=$ $(E+B G) Y D_{\alpha}$, where the columns of $Y$ are the eigenvectors. With $Z$ is nonsingular, $X$ is orthogonal and $\alpha_{j}^{2}+\beta_{j}^{2}=1(j=1, \cdots, n)$, we then have

$$
Z^{-1}(A+B F) X=D_{\alpha}+N_{\alpha}, \quad Z^{-1}(E+B G) X=D_{\beta}+N_{\beta} .
$$

Let $Q$ denote $Q_{2}$, then we arrive at:

\section{Optimization Problem 1.}

Given $A \in \mathbb{R}^{n \times n}, B \in \mathbb{R}^{n \times m},(A, B)$ is regular, $Q \in \mathbb{R}^{n \times(n-m)}$ is unitary and $Q^{\top} B=0$

$$
\begin{aligned}
& \min \left\|\left[N_{\alpha}, N_{\beta}\right]\right\|_{F}^{2} \\
& \text { s.t. }\left\{\begin{array}{l}
Q^{\top} A X-Q^{\top} Z D_{\alpha}-Q^{\top} Z N_{\alpha}=0 \quad N_{\alpha}, N_{\beta} \text { are } n \times n \text { strictly upper triangular, } \\
Q^{\top} E X-Q^{\top} Z D_{\beta}-Q^{\top} Z N_{\beta}=0, \quad X \text { is } n \times n \text { orthogonal, } Z \text { is nonsingular. } \\
X^{\top} X-I=0
\end{array}\right.
\end{aligned}
$$


Optimization Problem 1 is equivalent to:

$$
\begin{aligned}
& \min \operatorname{Vec}\left(N_{\alpha}\right)^{\top} \operatorname{Vec}\left(N_{\alpha}\right)+\operatorname{Vec}\left(N_{\beta}\right)^{\top} \operatorname{Vec}\left(N_{\beta}\right) \\
& \text { s.t. }\left\{\begin{array}{l}
\left(I \otimes Q^{\top} A\right) v(X)-\left(D_{\alpha}^{\top} \otimes Q^{\top}\right) v(Z)-\left(N_{\alpha}^{\top} \otimes Q^{\top}\right) v(Z)=0 \\
\left(I \otimes Q^{\top} E\right) v(X)-\left(D_{\beta}^{\top} \otimes Q^{\top}\right) v(Z)-\left(N_{\beta}^{\top} \otimes Q^{\top}\right) v(Z)=0 \\
d_{0}(X)^{\top} v(X)-\operatorname{Vec}(I)=0
\end{array}\right.
\end{aligned}
$$

where

$$
\begin{gathered}
N_{\alpha}=\left[\begin{array}{ccccc}
0 & \eta_{12} & \eta_{13} & \cdots & \eta_{1 n} \\
0 & 0 & \eta_{23} & \cdots & \eta_{2 n} \\
\vdots & \vdots & 0 & \ddots & \vdots \\
\vdots & \vdots & \vdots & \ddots & \eta_{n-1, n} \\
0 & 0 & 0 & \cdots & 0
\end{array}\right] \in \mathbb{R}^{n \times n}, \operatorname{Vec}\left(N_{\alpha}\right)=\left[\begin{array}{c}
\frac{\eta_{12}}{\eta_{13}} \\
\frac{\eta_{23}}{\vdots} \\
\frac{\eta_{1 n}}{\vdots} \\
\eta_{n-1, n}
\end{array}\right] \in \mathbb{R}^{n(n-1) / 2 \equiv p} \\
N_{\beta}=\left[\begin{array}{ccccc}
0 & \zeta_{12} & \zeta_{13} & \cdots & \zeta_{1 n} \\
0 & 0 & \zeta_{23} & \cdots & \zeta_{2 n} \\
\vdots & \vdots & 0 & \ddots & \vdots \\
\vdots & \vdots & \vdots & \ddots & \zeta_{n-1, n} \\
0 & 0 & 0 & \cdots & 0
\end{array}\right] \in \mathbb{R}^{n \times n}, \operatorname{Vec}\left(N_{\beta}\right)=\left[\begin{array}{c}
\frac{\zeta_{12}}{\zeta_{13}} \\
\frac{\zeta_{23}}{\vdots} \\
\frac{\zeta_{1 n}}{\vdots} \\
\zeta_{n-1, n}
\end{array}\right] \in \mathbb{R}^{p} .
\end{gathered}
$$

Here, we write $C^{k \times n}=\left[c_{1}, \cdots, c_{n}\right]$, so

$$
d_{0}(C)=\left[c_{1} \oplus\left[c_{1}, c_{2}\right] \oplus \cdots \oplus\left[c_{1}, \cdots, c_{n}\right]\right] \in \mathbb{R}^{k n \times q} .
$$

We then consider the Lagrangian function of Optimization Problem 1:

$$
\begin{aligned}
& L \\
& \quad\left(\gamma, \varepsilon, \delta, v(X), v(Z), \operatorname{Vec}\left(N_{\alpha}\right), \operatorname{Vec}\left(N_{\beta}\right)\right)=\operatorname{Vec}\left(N_{\alpha}\right)^{\top} \operatorname{Vec}\left(N_{\alpha}\right) \\
& \quad+\operatorname{Vec}\left(N_{\beta}\right)^{\top} \operatorname{Vec}\left(N_{\beta}\right)+\gamma^{\top}\left[\left(I \otimes Q^{\top} A\right) v(X)-\left(D_{\alpha}^{\top} \otimes Q^{\top}\right) v(Z)\right. \\
& \left.\quad-\left(N_{\alpha}^{\top} \otimes Q^{\top}\right) v(Z)\right]+\varepsilon^{\top}\left[\left(I \otimes Q^{\top} E\right) v(X)\right. \\
& \left.\quad-\left(D_{\beta}^{\top} \otimes Q^{\top}\right) v(Z)-\left(N_{\beta}^{\top} \otimes Q^{\top}\right) v(Z)\right]+\delta^{\top}\left[d_{0}(X)^{\top} v(X)-\operatorname{Vec}(I)\right]
\end{aligned}
$$


where

$$
\begin{aligned}
& \gamma=[\underbrace{\gamma_{1}^{\top}}_{l}|\underbrace{\gamma_{2}^{\top}}_{l}| \cdots \mid \underbrace{\gamma_{n}^{\top}}_{l}]^{\top} \in \mathbb{R}^{l n}, \\
& R=\left[\gamma_{1}, \gamma_{2}, \cdots, \gamma_{n}\right], \\
& \varepsilon=[\underbrace{\varepsilon_{1}^{\top}}_{l}|\underbrace{\varepsilon_{2}^{\top}}_{l}| \cdots \mid \underbrace{\varepsilon_{n}^{\top}}_{l}]^{\top} \in \mathbb{R}^{l n}, \\
& W=\left[\varepsilon_{1}, \varepsilon_{2}, \cdots, \varepsilon_{n}\right], \\
& \delta=[\underbrace{\delta_{1}^{\top}}_{1}|\underbrace{\delta_{2}^{\top}}_{2}| \cdots \mid \underbrace{\delta_{n}^{\top}}_{n}]^{\top}=\left[\delta_{11}\left|\delta_{21}, \delta_{22}\right| \cdots \mid \delta_{n 1}, \delta_{n 2}, \cdots, \delta_{n n}\right]^{\top} \in \mathbb{R}^{q} .
\end{aligned}
$$

The derivatives of $L$ satisfy

(8) $\quad f_{1} \equiv \frac{\partial L}{\partial \gamma}=\left(I \otimes Q^{\top} A\right) v(X)-\left(D_{\alpha}^{\top} \otimes Q^{\top}\right) v(Z)-\left(N_{\alpha}^{\top} \otimes Q^{\top}\right) v(Z)=0$,

(9) $\quad f_{2} \equiv \frac{\partial L}{\partial \varepsilon}=\left(I \otimes Q^{\top} E\right) v(X)-\left(D_{\beta}^{\top} \otimes Q^{\top}\right) v(Z)-\left(N_{\beta}^{\top} \otimes Q^{\top}\right) v(Z)=0$,

$$
\begin{gathered}
f_{3} \equiv \frac{\partial L}{\partial \delta}=d_{0}(X)^{\top} v(X)-\operatorname{Vec}(I)=0, \\
\text { (11) } f_{4} \equiv \frac{\partial L}{\partial v(X)}=\left(I \otimes A^{\top} Q\right) \gamma+\left(I \otimes E^{\top} Q\right) \varepsilon+v(X \Delta)=0, \\
\text { (12) } f_{5} \equiv \frac{\partial L}{\partial v(Z)}=-\left[\left(D_{\alpha} \otimes Q\right)+\left(N_{\alpha} \otimes Q\right)\right] \gamma-\left[\left(D_{\beta} \otimes Q\right)+\left(N_{\beta} \otimes Q\right)\right] \varepsilon=0, \\
f_{6} \equiv \frac{\partial L}{\partial \operatorname{Vec}\left(N_{\alpha}\right)}=2 \operatorname{Vec}\left(N_{\alpha}\right)-d_{1}\left(Q^{\top} Z\right)^{\top} \gamma=0,
\end{gathered}
$$

$$
f_{7} \equiv \frac{\partial L}{\partial \operatorname{Vec}\left(N_{\beta}\right)}=2 \operatorname{Vec}\left(N_{\beta}\right)-d_{1}\left(Q^{\top} Z\right)^{\top} \varepsilon=0
$$

where

$$
\Delta=\left[\begin{array}{ccccc}
2 \delta_{11} & \delta_{21} & \delta_{31} & \cdots & \delta_{n 1} \\
\delta_{21} & 2 \delta_{22} & \delta_{32} & \cdots & \delta_{n 2} \\
\vdots & \vdots & 2 \delta_{33} & \cdots & \vdots \\
\vdots & \vdots & \vdots & \cdots & \delta_{n, n-1} \\
\delta_{n 1} & \delta_{n 2} & \delta_{n 3} & \cdots & 2 \delta_{n n}
\end{array}\right]
$$


We can apply Newton's method to $f \equiv\left(f_{1}^{\top}, f_{2}^{\top}, \cdots, f_{7}^{\top}\right)^{\top}$, which can be formulated as

$$
\left[\begin{array}{c}
\gamma \\
\varepsilon \\
\delta \\
v(X) \\
v(Z) \\
\operatorname{Vec}\left(N_{\alpha}\right) \\
\operatorname{Vec}\left(N_{\beta}\right)
\end{array}\right]_{\text {new }}=\left[\begin{array}{c}
\gamma \\
\varepsilon \\
\delta \\
v(X) \\
v(Z) \\
\operatorname{Vec}\left(N_{\alpha}\right) \\
\operatorname{Vec}\left(N_{\beta}\right)
\end{array}\right]-J_{f}^{-1}\left[\begin{array}{l}
f_{1} \\
f_{2} \\
f_{3} \\
f_{4} \\
f_{5} \\
f_{6} \\
f_{7}
\end{array}\right]
$$

where the symmetric

$$
\begin{gathered}
J_{f}=\left[\begin{array}{ccccccc}
\frac{\partial f_{1}}{\partial \gamma} & \frac{\partial f_{1}}{\partial \varepsilon} & \frac{\partial f_{1}}{\partial \delta} & \frac{\partial f_{1}}{\partial v(X)} & \frac{\partial f_{1}}{\partial v(Z)} & \frac{\partial f_{1}}{\partial \operatorname{Vec}\left(N_{\alpha}\right)} & \frac{\partial f_{1}}{\partial \operatorname{Vec}\left(N_{\beta}\right)} \\
\vdots & \vdots & \vdots & \vdots & \vdots & \vdots & \vdots \\
\frac{\partial f_{7}}{\partial \gamma} & \frac{\partial f_{7}}{\partial \varepsilon} & \frac{\partial f_{7}}{\partial \delta} & \frac{\partial f_{7}}{\partial v(X)} & \frac{\partial f_{7}}{\partial v(Z)} & \frac{\partial f_{7}}{\partial \operatorname{Vec}\left(N_{\alpha}\right)} & \frac{\partial f_{7}}{\partial \operatorname{Vec}\left(N_{\beta}\right)}
\end{array}\right]= \\
{\left[\begin{array}{cccccccc}
0 & 0 & 0 & I \otimes Q^{\top} A & -D_{\alpha}^{\top} \otimes Q^{\top}-N_{\alpha}^{\top} \otimes Q^{\top} & -d_{1}\left(Q^{\top} Z\right) & 0 \\
0 & 0 & 0 & I \otimes Q^{\top} E & -D_{\beta}^{\top} \otimes Q^{\top}-N_{\beta}^{\top} \otimes Q^{\top} & 0 & -d_{1}\left(Q^{\top} Z\right) \\
* & 0 & 0 & d_{0}(X)^{\top}+d_{2}\left(X^{\top}\right) & 0 & 0 & 0 \\
* & * & * & \Delta \otimes I & 0 & 0 & 0 \\
* & * & * & * & 0 & -d_{3}\left(Q\left[\gamma_{2}, \cdots, \gamma_{n}\right]\right) & -d_{3}\left(Q\left[\varepsilon_{2}, \cdots, \varepsilon_{n}\right]\right) \\
* & * & * & * & * & 0 & 0 \\
* & * & * & * & * & 0 & 2 I_{p}
\end{array}\right]}
\end{gathered}
$$

and

$$
\begin{aligned}
& d_{1}(C)=\left[\begin{array}{cccccc}
0 & \cdots & \cdots & \cdots & \cdots & 0 \\
c_{1} \oplus\left[c_{1}, c_{2}\right] & \oplus & \cdots & \left.\oplus c_{1}, \ldots, c_{n-1}\right]
\end{array}\right] \in \mathbb{R}^{k n \times p}, \\
& d_{2}\left(C^{\top}\right)=\left[\begin{array}{l}
c_{1}^{\top} \\
c_{2}^{\top} \oplus c_{2}^{\top} \\
c_{3}^{\top} \oplus c_{3}^{\top} \oplus c_{3}^{\top} \\
\vdots \\
c_{n}^{\top} \oplus \cdots \oplus c_{n}^{\top}
\end{array}\right] \in \mathbb{R}^{q \times k n}, \\
& d_{3}(C)=k\left\{\left[\begin{array}{ccccc}
c_{2} & c_{3} \oplus c_{3} & c_{4} \oplus c_{4} \oplus c_{4} & \cdots & c_{n} \oplus \cdots \oplus c_{n} \\
0 & \cdots & \cdots & \cdots & 0
\end{array}\right] \in \mathbb{R}^{k n \times p} .\right.
\end{aligned}
$$

Applying Newton's method to Optimization Problem 1, we obtain $Z, X$ then by using (4), the feedback matrices $F, G$. Now, we can write down the Schur-Newton Algorithm for the RPAP_DS with real eigenvalues: 


\section{Algorithm 1 (Real Schur-Newton).}

(1) Use the Schur algorithm in Section 3 to find an initial $X_{0}, Z_{0}$ and $N_{\alpha 0}, N_{\beta 0}$.

(2) Substitute $X_{0}, Z_{0}, N_{\alpha 0}, N_{\beta 0}$ into (11)-(14), producing an over-determined linear system for $\left(\gamma_{0}^{\top}, \varepsilon_{0}^{\top}, \delta_{0}^{\top}\right)^{\top}$ :

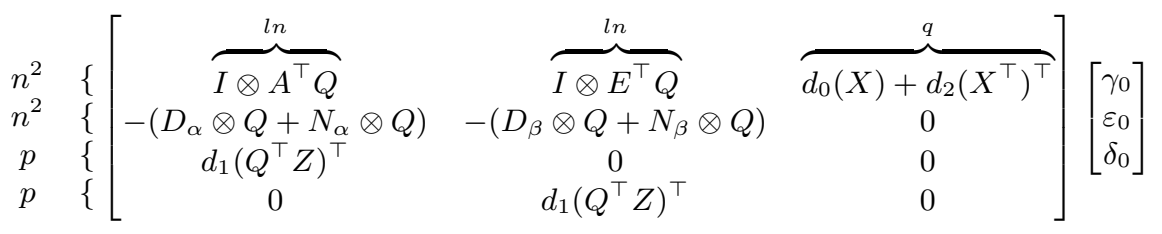

$$
\begin{aligned}
& =\left[\begin{array}{c}
0 \\
0 \\
2 \operatorname{Vec}\left(N_{\alpha}\right) \\
2 \operatorname{Vec}\left(N_{\beta}\right)
\end{array}\right]
\end{aligned}
$$

where $l=n-m, p, q$ are defined as before and $n^{2}+p \geq l n+q$. Use the least squares method to solve the over-determined system in (??) for $\gamma_{0}, \varepsilon_{0}$ and $\delta_{0}$.

(3) Choose $\left\{\gamma_{0}, \varepsilon_{0}, \delta_{0}, v\left(X_{0}\right), v\left(Z_{0}\right), \operatorname{Vec}\left(N_{\alpha 0}\right), \operatorname{Vec}\left(N_{\beta 0}\right)\right\}$ to be the starting values, run Newton's iteration (15) until convergence to $X, Z$ and $N_{\alpha}, N_{\beta}$.

(4) Substitute the $X, Z$ and $N_{\alpha}, N_{\beta}$ into (??) to obtain the feedback matrices $F, G$.

\subsection{Complex Eigenvalues}

Let $\left\{\left(\alpha_{1}, \beta_{1}\right), \cdots,\left(\alpha_{n-2 s}, \beta_{n-2 s}\right) ;\left(\mu_{1} \pm i \nu_{1}, \kappa_{1} \pm i \tau_{1}\right), \cdots,\left(\mu_{s} \pm i \nu_{s}, \kappa_{s} \pm i \tau_{s}\right)\right\}$ be the prescribed eigenvalues, where $s$ is the number of complex eigenvalue pairs. As in the real eigenvalue case, we seek feedback matrices $F, G \in \mathbb{R}^{n \times n}$ such that

$$
\lambda(A+B F, E+B G)=\lambda\left\{\left[\left(\begin{array}{cc}
\Lambda & 0 \\
0 & I
\end{array}\right),\left(\begin{array}{ll}
I & 0 \\
0 & 0
\end{array}\right)\right]\right\}=\lambda\left(D_{\alpha}, D_{\beta}\right)
$$

where $\alpha_{j}, \beta_{j}, \mu_{l}, \nu_{l}, \kappa_{l}, \tau_{l} \in \mathbb{R}, \alpha_{j}^{2}+\beta_{j}^{2}=1=\mu_{l}^{2}+\nu_{l}^{2}+\kappa_{l}^{2}+\tau_{l}^{2}(j=1, \cdots, n-$ $2 s ; l=1, \cdots, s)$, and $D_{\alpha} \equiv \operatorname{diag}\left\{\alpha_{1}, \cdots, \alpha_{n-2 s} ; \mu_{1} \pm i \nu_{1}, \cdots, \mu_{s} \pm i \nu_{s}\right\}, D_{\beta} \equiv$ $\operatorname{diag}\left\{\beta_{1}, \cdots, \beta_{n-2 s} ; \kappa_{1} \pm i \tau_{1}, \cdots, \kappa_{s} \pm i \tau_{s}\right\}$. With a nonsingular $Z$ and orthogonal $X$, we require

$$
Z^{-1}(A+B F) X=D_{\alpha}+N_{\alpha}, \quad Z^{-1}(E+B G) X=D_{\beta}+N_{\beta},
$$

where

$$
D_{\alpha}=\left[\alpha_{1} \oplus \cdots \oplus \alpha_{n-2 s} \oplus\left[\begin{array}{ll}
a_{1} & b_{2} \\
b_{1} & a_{2}
\end{array}\right] \oplus \cdots \oplus\left[\begin{array}{ll}
a_{2 s-1} & b_{2 s} \\
b_{2 s-1} & a_{2 s}
\end{array}\right]\right]
$$


with

$$
\begin{aligned}
& \left\{\begin{array}{l}
a_{1}, \cdots, a_{2 s}, b_{1}, \cdots, b_{2 s} \in \mathbb{R}, \\
a_{2 j-1}+a_{2 j}=2 \mu_{j}, j=1, \cdots, s ; \\
a_{2 j-1} a_{2 j}-b_{2 j-1} b_{2 j}=\mu_{j}^{2}+\nu_{j}^{2}, j=1, \cdots, s .
\end{array},\right. \\
& D_{\beta}=\left[\beta_{1} \oplus \cdots \oplus \beta_{n-2 s} \oplus\left[\begin{array}{ll}
c_{1} & d_{2} \\
d_{1} & c_{2}
\end{array}\right] \oplus \cdots \oplus\left[\begin{array}{ll}
c_{2 s-1} & d_{2 s} \\
d_{2 s-1} & c_{2 s}
\end{array}\right]\right], \\
& \left\{\begin{array}{l}
c_{1}, \cdots, c_{2 s}, d_{1}, \cdots, d_{2 s} \in \mathbb{R}, \\
c_{2 j-1}+c_{2 j}=2 \kappa_{j}, j=1, \cdots, s ; \\
c_{2 j-1} c_{2 j}-d_{2 j-1} d_{2 j}=\kappa_{j}^{2}+\tau_{j}^{2}, j=1, \cdots, s .
\end{array},\right. \\
& \begin{array}{ccccccccc}
\eta_{2} & \eta_{3} & \cdots & \eta_{n-2 s} & \eta_{n-2 s+1} & \eta_{n-2 s+2} & \cdots & \eta_{n-1} & \eta_{n} \\
\downarrow & \downarrow & & \downarrow & \downarrow & \downarrow & & \downarrow & \downarrow
\end{array}
\end{aligned}
$$

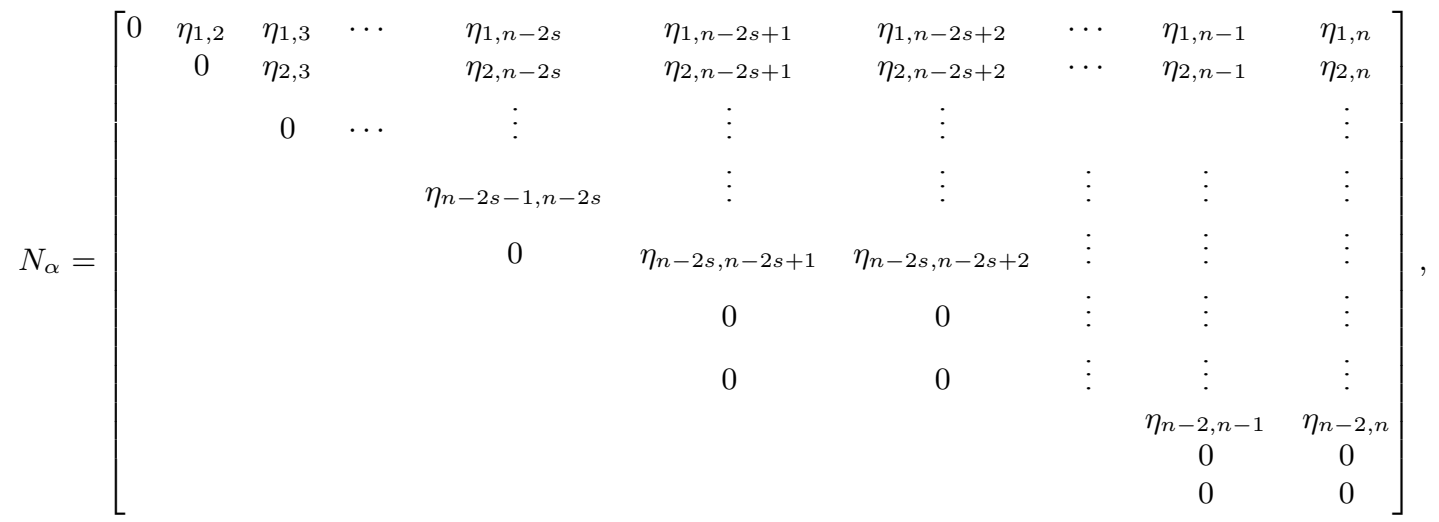

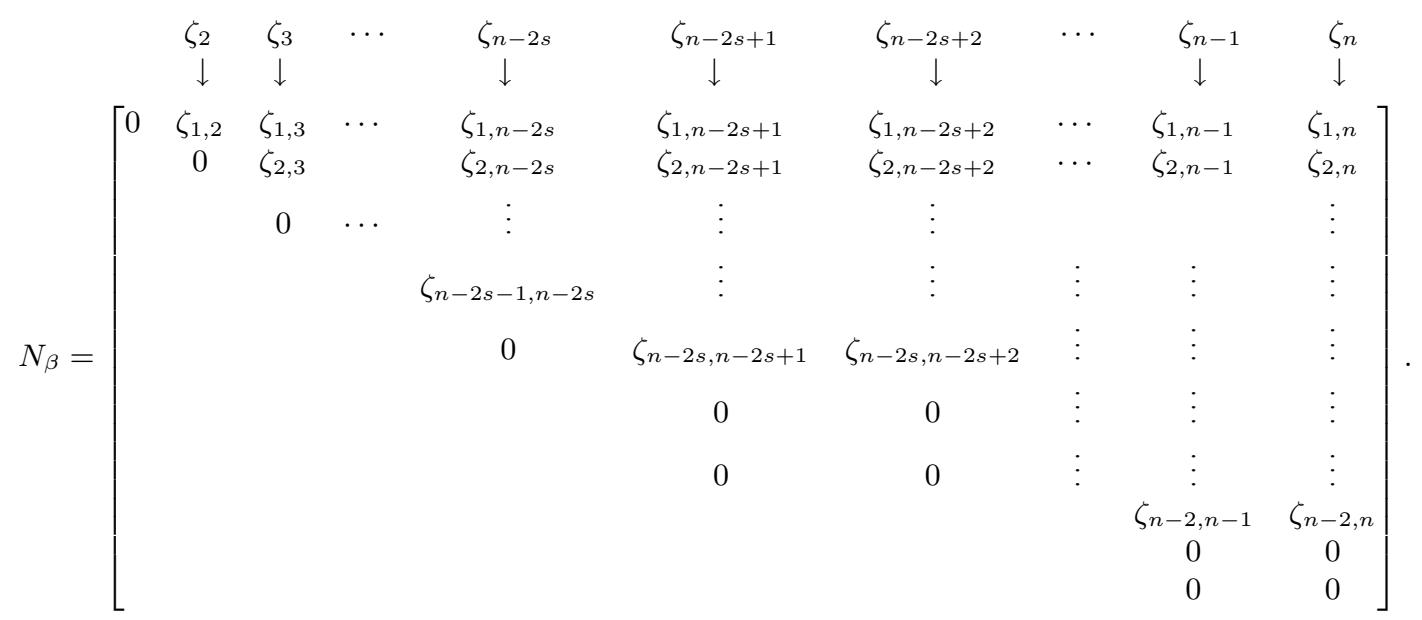

We arrive at the optimization problem for complex eigenvalues:

Optimization Problem 2.

Given $A \in \mathbb{R}^{n \times n}, B \in \mathbb{R}^{n \times m},(A, B)$ regular, $Q \in \mathbb{R}^{n \times(n-m)}$ is orthogonal and 
$Q^{\top} B=0 ;$

$$
\min \left\|\left[N_{\alpha}, N_{\beta}\right]\right\|_{F}^{2}
$$

s.t. $\begin{cases}Q^{\top} A X-Q^{\top} Z\left(D_{\alpha}+N_{\alpha}\right)=0 & D_{\alpha}, D_{\beta}, N_{\alpha}, N_{\beta} \text { are as defined before, } \\ Q^{\top} E X-Q^{\top} Z\left(D_{\beta}+N_{\beta}\right)=0 & X \text { is } n \times n \text { orthogonal, } Z \text { is } n \times n \text { nonsingular. } \\ X^{\top} X-I=0 & \end{cases}$

Optimization Problem 2 is equivalent to:

$$
\begin{gathered}
\min \operatorname{Vec}\left(N_{\alpha}\right)^{\top} \operatorname{Vec}\left(N_{\alpha}\right)+\operatorname{Vec}\left(N_{\beta}\right)^{\top} \operatorname{Vec}\left(N_{\beta}\right) \quad \text { s.t. } \\
\left(I \otimes Q^{\top} A\right) v(X)-\left(D_{\alpha}^{\top} \otimes Q^{\top}\right) v(Z)-\left(N_{\alpha}^{\top} \otimes Q^{\top}\right) v(Z)=0 \\
\left(I \otimes Q^{\top} E\right) v(X)-\left(D_{\beta}^{\top} \otimes Q^{\top}\right) v(Z)-\left(N_{\beta}^{\top} \otimes Q^{\top}\right) v(Z)=0 \\
d_{0}(X)^{\top} v(X)-\operatorname{Vec}(I)=0 \\
{\left[\begin{array}{c}
a_{1}+a_{2}-2 \mu_{1}=0 \\
\vdots \\
a_{2 s-1}+a_{2 s}-2 \mu_{s}=0
\end{array}\right]} \\
{\left[\begin{array}{c}
c_{1}+c_{2}-2 \kappa_{1}=0 \\
\vdots \\
c_{2 s-1}+c_{2 s}-2 \kappa_{s}=0
\end{array}\right]} \\
{\left[\begin{array}{c}
a_{1} a_{2}-b_{1} b_{2}-\left(\mu_{1}^{2}+\nu_{1}^{2}\right)=0 \\
\vdots \\
a_{2 s-1} a_{2 s}-b_{2 s-1} b_{2 s}-\left(\mu_{s}^{2}+\nu_{s}^{2}\right)=0
\end{array}\right]} \\
{\left[\begin{array}{c}
c_{1} c_{2}-d_{1} d_{2}-\left(\kappa_{1}^{2}+\tau_{1}^{2}\right)=0 \\
\vdots \\
c_{2 s-1} c_{2 s}-d_{2 s-1} d_{2 s}-\left(\kappa_{s}^{2}+\tau_{s}^{2}\right)=0
\end{array}\right]}
\end{gathered}
$$

for which the Lagrangian function equals

$$
\begin{aligned}
& L\left(\gamma, \varepsilon, \delta, \omega, \theta, \xi, \sigma, v(X), v(Z), a, b, c, d, \operatorname{Vec}\left(N_{\alpha}\right), \operatorname{Vec}\left(N_{\beta}\right)\right) \\
= & \operatorname{Vec}\left(N_{\alpha}\right)^{\top} \operatorname{Vec}\left(N_{\alpha}\right)+\operatorname{Vec}\left(N_{\beta}\right)^{\top} \operatorname{Vec}\left(N_{\beta}\right) \\
& +\gamma^{\top}\left[\left(I \otimes Q^{\top} A\right) v(X)-\left(D_{\alpha}^{\top} \otimes Q^{\top}\right) v(Z)-\left(N_{\alpha}^{\top} \otimes Q^{\top}\right) v(Z)\right] \\
& +\varepsilon^{\top}\left[\left(I \otimes Q^{\top} E\right) v(X)-\left(D_{\beta}^{\top} \otimes Q^{\top}\right) v(Z)-\left(N_{\beta}^{\top} \otimes Q^{\top}\right) v(Z)\right] \\
& +\delta^{\top}\left[d_{0}(X)^{\top} v(X)-\operatorname{Vec}(I)\right]+\sum_{j=1}^{s} \omega_{j}\left(a_{2 j-1}\right.
\end{aligned}
$$




$$
\begin{aligned}
& \left.+a_{2 j}-2 \mu_{j}\right)+\sum_{j=1}^{s} \theta_{j}\left(c_{2 j-1}+c_{2 j}-2 \kappa_{j}\right) \\
& +\sum_{\substack{j=1 \\
s}}^{s} \xi_{j}\left[a_{2 j-1} a_{2 j}-b_{2 j-1} b_{2 j}-\left(\mu_{j}^{2}+\nu_{j}^{2}\right)\right] \\
& +\sum_{j=1}^{s} \sigma_{j}\left[c_{2 j-1} c_{2 j}-d_{2 j-1} d_{2 j}-\left(\kappa_{j}^{2}+\tau_{j}^{2}\right)\right]
\end{aligned}
$$

where

$$
\begin{array}{ll}
\omega=\left(\omega_{1}, \omega_{2}, \cdots, \omega_{s}\right)^{\top}, & \theta=\left(\theta_{1}, \theta_{2}, \cdots, \theta_{s}\right)^{\top}, \\
\xi=\left(\xi_{1}, \xi_{2}, \cdots, \xi_{s}\right)^{\top}, & \sigma=\left(\sigma_{1}, \sigma_{2}, \cdots, \sigma_{s}\right)^{\top}, \\
a=\left(a_{1}, a_{2}, \cdots, a_{2 s}\right)^{\top}, & b=\left(b_{1}, b_{2}, \cdots, b_{2 s}\right)^{\top}, \\
c=\left(c_{1}, c_{2}, \cdots, c_{2 s}\right)^{\top}, & d=\left(d_{1}, d_{2}, \cdots, d_{2 s}\right)^{\top} .
\end{array}
$$

The derivatives of $L$ are

(17) $f_{1} \equiv \frac{\partial L}{\partial \gamma}=\left(I \otimes Q^{\top} A\right) v(X)-\left(D_{\alpha}^{\top} \otimes Q^{\top}\right) v(Z)-\left(N_{\alpha}^{\top} \otimes Q^{\top}\right) v(Z)=0$

(18) $f_{2} \equiv \frac{\partial L}{\partial \varepsilon}=\left(I \otimes Q^{\top} E\right) v(X)-\left(D_{\beta}^{\top} \otimes Q^{\top}\right) v(Z)-\left(N_{\beta}^{\top} \otimes Q^{\top}\right) v(Z)=0$,

$$
f_{3} \equiv \frac{\partial L}{\partial \delta}=d_{0}(X)^{\top} v(X)-\operatorname{Vec}(I)=0
$$

$$
f_{4} \equiv \frac{\partial L}{\partial \omega}=\left[\begin{array}{c}
a_{1}+a_{2}-2 \mu_{1} \\
\vdots \\
a_{2 s-1}+a_{2 s}-2 \mu_{s}
\end{array}\right]
$$

$$
f_{5} \equiv \frac{\partial L}{\partial \theta}=\left[\begin{array}{c}
c_{1}+c_{2}-2 \kappa_{1} \\
\vdots \\
c_{2 s-1}+c_{2 s}-2 \kappa_{s}
\end{array}\right]
$$

$f_{6} \equiv \frac{\partial L}{\partial \xi}=\left[\begin{array}{c}a_{1} a_{2}-b_{1} b_{2}-\left(\mu_{1}^{2}+\nu_{1}^{2}\right)=0 \\ \vdots \\ a_{2 s-1} a_{2 s}-b_{2 s-1} b_{2 s}-\left(\mu_{s}^{2}+\nu_{s}^{2}\right)=0\end{array}\right]$,

$$
f_{7} \equiv \frac{\partial L}{\partial \sigma}=\left[\begin{array}{c}
c_{1} c_{2}-d_{1} d_{2}-\left(\kappa_{1}^{2}+\tau_{1}^{2}\right)=0 \\
\vdots \\
c_{2 s-1} c_{2 s}-d_{2 s-1} d_{2 s}-\left(\kappa_{s}^{2}+\tau_{s}^{2}\right)=0
\end{array}\right] \text {, }
$$

$$
f_{8} \equiv \frac{\partial L}{\partial v(X)}=\left(I \otimes A^{\top} Q\right) \gamma+\left(I \otimes E^{\top} Q\right) \varepsilon+v(X \Delta)=0
$$

(25) $f_{9} \equiv \frac{\partial L}{\partial v(Z)}=-\left[\left(D_{\alpha} \otimes Q\right)+\left(N_{\alpha} \otimes Q\right)\right] \gamma-\left[\left(D_{\beta} \otimes Q\right)+\left(N_{\beta} \otimes Q\right)\right] \varepsilon=0$, 
(26)

$$
\begin{aligned}
& f_{10} \equiv \frac{\partial L}{\partial a}=\left[\begin{array}{c}
\omega_{1} \\
\omega_{1} \\
\vdots \\
\omega_{s} \\
\omega_{s}
\end{array}\right]+\left[\begin{array}{c}
\xi_{1} a_{2} \\
\xi_{1} a_{1} \\
\vdots \\
\xi_{s} a_{2 s} \\
\xi_{s} a_{2 s-1}
\end{array}\right]-\left[\begin{array}{ccc}
\gamma_{n-2 s+1}^{\top} & & \\
& \ddots & \\
& & \gamma_{n}^{\top}
\end{array}\right] v\left(Q^{\top}\left[z_{n-2 s+1}, \cdots, z_{n}\right]\right)=0, \\
& f_{11} \equiv \frac{\partial L}{\partial b}=-\left[\begin{array}{c}
\xi_{1} b_{2} \\
\xi_{1} b_{1} \\
\vdots \\
\xi_{s} b_{2 s} \\
\xi_{s} b_{2 s-1}
\end{array}\right]-\left[\begin{array}{lll}
\gamma_{n-2 s+1}^{\top} & & \\
& \ddots & \\
& & \gamma_{n}^{\top}
\end{array}\right] \Pi_{s} v\left(Q^{\top}\left[z_{n-2 s+1}, \cdots, z_{n}\right]\right)=0,
\end{aligned}
$$

$$
f_{12} \equiv \frac{\partial L}{\partial c}=\left[\begin{array}{c}
\theta_{1} \\
\theta_{1} \\
\vdots \\
\theta_{s} \\
\theta_{s}
\end{array}\right]+\left[\begin{array}{c}
\sigma_{1} c_{2} \\
\sigma_{1} c_{1} \\
\vdots \\
\sigma_{s} c_{2 s} \\
\sigma_{s} c_{2 s-1}
\end{array}\right]-\left[\begin{array}{ccc}
\varepsilon_{n-2 s+1}^{\top} & & \\
& \ddots & \\
& & \varepsilon_{n}^{\top}
\end{array}\right] v\left(Q^{\top}\left[z_{n-2 s+1}, \cdots, z_{n}\right]\right)=0 \text {, }
$$

$$
\begin{aligned}
& f_{13} \equiv \frac{\partial L}{\partial d}=-\left[\begin{array}{c}
\sigma_{1} d_{2} \\
\sigma_{1} d_{1} \\
\vdots \\
\sigma_{s} d_{2 s} \\
\sigma_{s} d_{2 s-1}
\end{array}\right]-\left[\begin{array}{lll}
\varepsilon_{n-2 s+1}^{\top} & & \\
& \ddots & \\
& & \varepsilon_{n}^{\top}
\end{array}\right] \Pi_{s} v\left(Q^{\top}\left[z_{n-2 s+1}, \cdots, z_{n}\right]\right)=0, \\
& f_{14} \equiv \frac{\partial L}{\partial \operatorname{Vec}\left(N_{\alpha}\right)}=2 \operatorname{Vec}\left(N_{\alpha}\right)-\widehat{d_{1}}\left(Q^{\top} Z, s\right)^{\top} \gamma=0, \\
& \text { (31) } f_{15} \equiv \frac{\partial L}{\partial \operatorname{Vec}\left(N_{\beta}\right)}=2 \operatorname{Vec}\left(N_{\beta}\right)-\widehat{d}_{1}\left(Q^{\top} Z, s\right)^{\top} \varepsilon=0
\end{aligned}
$$

where $\widehat{d_{1}}(C, s)=$

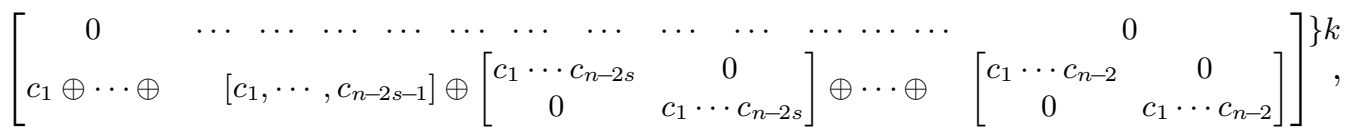

$$
\begin{aligned}
& \Pi_{s}=\underbrace{\left[\begin{array}{ll}
0 & I \\
I & 0
\end{array}\right] \oplus \cdots \oplus\left[\begin{array}{ll}
0 & I \\
I & 0
\end{array}\right]}_{s} .
\end{aligned}
$$

We can then obtain the symmetric gradient matrix of $f \equiv\left[f_{1}^{\top}, f_{2}^{\top}, \cdots, f_{15}^{\top}\right]^{\top}$ :

$$
J_{f}=\left[\begin{array}{ll}
J_{1} & J_{2} \\
J_{3} & J_{4}
\end{array}\right]
$$


where $J_{1}=0, J_{3}=J_{2}^{\top}$,

$$
J_{2}=\left[\begin{array}{cccccccc}
\Omega_{1} & \Phi_{1} & -\widehat{d_{4}}\left(Q^{\top} Z, s\right) & -d_{4}\left(Q^{\top} Z, s\right) & 0 & 0 & -\widehat{d_{1}}\left(Q^{\top} Z, s\right) & 0 \\
\Omega_{2} & \Phi_{2} & 0 & 0 & -\widehat{d_{4}}\left(Q^{\top} Z, s\right) & -d_{4}\left(Q^{\top} Z, s\right) & 0 & -\widehat{d_{1}}\left(Q^{\top} Z, s\right) \\
\Xi & 0 & 0 & 0 & 0 & 0 & 0 & 0 \\
0 & 0 & d_{5}(e) & 0 & 0 & 0 & 0 & 0 \\
0 & 0 & 0 & 0 & d_{5}(e) & 0 & 0 & 0 \\
0 & 0 & d_{5}(a) & -d_{5}(b) & 0 & 0 & 0 & 0 \\
0 & 0 & 0 & 0 & d_{5}(c) & -d_{5}(d) & 0 & 0
\end{array}\right]
$$

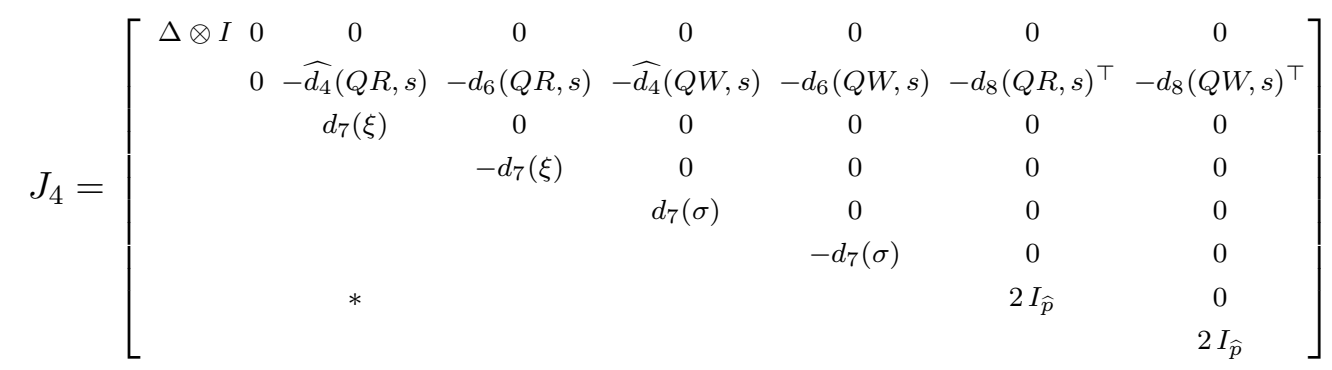

with

$$
\begin{aligned}
& \Omega_{1}=I \otimes Q^{\top} A, \quad \Omega_{2}=I \otimes Q^{\top} E, \\
& \Phi_{1}=-D_{\alpha}^{\top} \otimes Q^{\top}-N_{\alpha}^{\top} \otimes Q^{\top}, \quad \Phi_{2}=-D_{\beta}^{\top} \otimes Q^{\top}-N_{\beta}^{\top} \otimes Q^{\top}, \\
& \widehat{p}=(n-2 s)(n-2 s-1) / 2+2 s(n-s-1) \text {, } \\
& e=[\underbrace{1,1, \cdots, 1}_{2 s}]^{\top}, \quad \Xi=d_{0}(X)^{\top}+d_{2}\left(X^{\top}\right), \\
& d_{4}(C, s)=\left[\begin{array}{cccc}
0 & \cdots & \cdots & 0 \\
\vdots & & & \vdots \\
0 & \cdots & \cdots & 0 \\
{\left[\begin{array}{cc}
c_{n-2 s+2} & \\
& c_{n-2 s+1}
\end{array}\right]} & \left.\left.\oplus \cdots \oplus\left[\begin{array}{cc}
c_{n} & \\
& c_{n-1}
\end{array}\right]\right]\right\} & k(2 s)
\end{array}\right. \\
& \left.\widehat{d}_{4}(C, s)=\left[\begin{array}{ccccccc}
0 & \cdots & \cdots & \cdots & \cdots & \cdots & 0 \\
\vdots & & & & & & \vdots \\
0 & \cdots & \cdots & \cdots & \cdots & \cdots & 0 \\
c_{n-2 s+1} & \oplus & c_{n-2 s+2} & \oplus \cdots \oplus & c_{n-1} & \oplus & c_{n}
\end{array}\right]\right\} \quad k(n-2 s) \\
& \left.d_{5}(\psi) \equiv d_{5}\left(\left[\psi_{1}, \cdots, \psi_{2 s}\right]^{\top}\right)\right)=\left[\psi_{2}, \psi_{1}\right] \oplus\left[\psi_{4}, \psi_{3}\right] \oplus \cdots \oplus\left[\psi_{2 s}, \psi_{2 s-1}\right],
\end{aligned}
$$




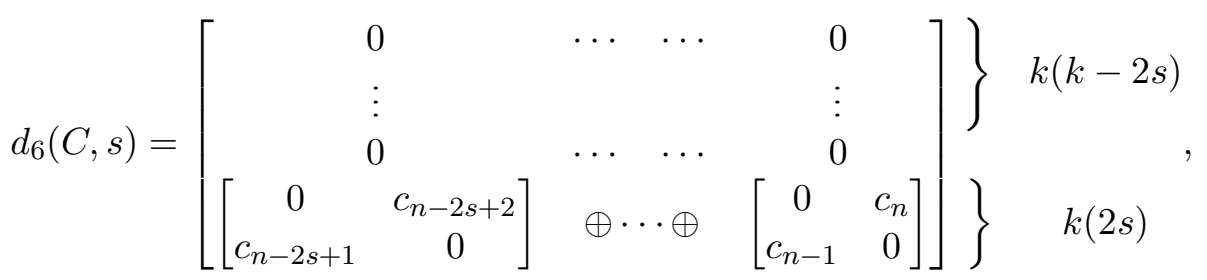

$$
\begin{aligned}
& \left.d_{7}(\psi) \equiv d_{7}\left(\left[\psi_{1}, \cdots, \psi_{2 s}\right]^{\top}\right)\right)=\left[\begin{array}{cc}
0 & \psi_{1} \\
\psi_{1} & 0
\end{array}\right] \oplus\left[\begin{array}{cc}
0 & \psi_{2} \\
\psi_{2} & 0
\end{array}\right] \oplus \cdots \oplus\left[\begin{array}{cc}
0 & \psi_{s} \\
\psi_{s} & 0
\end{array}\right],
\end{aligned}
$$

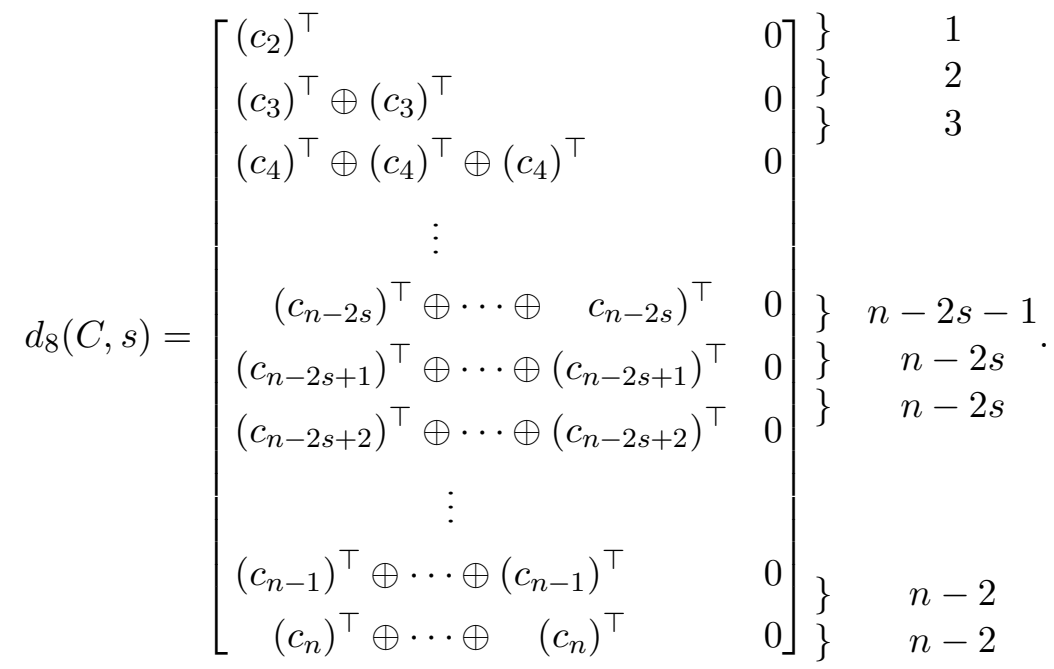

Similar to Algorithm 1, we solve Optimization Problem 2 by Newton's iteration for the real $F, G$ :

\section{Algorithm 2 (Complex Schur-Newton).}

(1) Use the Schur method in Section 3 to find the initial $X_{0}, Z_{0}$ and $N_{\alpha 0}, N_{\beta 0}$.

(2) Substitute $X_{0}, Z_{0}, N_{\alpha 0}, N_{\beta 0}$ into (24)-(31), we obtain $\left\{\gamma_{0}, \varepsilon_{0}, \delta_{0}, \omega_{0}, \theta_{0}, \xi_{0}, \sigma_{0}\right.$, $\left.a_{0}, b_{0}, c_{0}, d_{0}\right\}$.

(3) With $\left\{\gamma_{0}, \varepsilon_{0}, \delta_{0}, \omega_{0}, \theta_{0}, \xi_{0}, \sigma_{0}, a_{0}, b_{0}, c_{0}, d_{0}, v\left(X_{0}\right), v\left(Z_{0}\right), \operatorname{Vec}\left(N_{\alpha 0}\right), \operatorname{Vec}\left(N_{\beta 0}\right)\right\}$ as starting values, run Newton's iteration until convergence to $X, Z$ and $N_{\alpha}$, $N_{\beta}$.

(4) Substitute $X, Z$ and $N_{\alpha}, N_{\beta}$ into (4) to obtain the feedback matrices $F, G$.

\section{Remark.}

- At Step 2, we set $a, b, c, d$ being the same as the given eigenvalues, obtaining $\gamma_{0}$ by substituting $Z_{0}, N_{\alpha 0}$ into (30) and $\varepsilon_{0}$ by substituting $Z_{0}, N_{\beta 0}$ into (31). Then from (24)-(29), we obtain $\delta_{0}, \omega_{0}, \theta_{0}, \xi_{0}$ and $\sigma_{0}$. 
- The starting point $\left\{\gamma_{0}, \varepsilon_{0}, \delta_{0}, \omega_{0}, \theta_{0}, \xi_{0}, \sigma_{0}, a_{0}, b_{0}, c_{0}, d_{0}, v\left(X_{0}\right), v\left(Z_{0}\right), \operatorname{Vec}\left(N_{\alpha 0}\right)\right.$, $\left.\operatorname{Vec}\left(N_{\beta 0}\right)\right\}$ is often far away from being optimal. In such an event, we apply the GBB Gradient method [13] to decrease the objective function sufficiently, before Newton's iteration is applied.

- At Step 4, since the matrix $X$ is orthogonal, we can use $X^{\top}$ in place of $X^{-1}$.

These remarks also hold for Algorithm 1.

\section{NUMERICAL EXAMPLES}

Algorithms 1 and 2 are applied to three examples, all with singular Es. The convergence tolerance is $10^{-8}$. The numerical computations were carried out on a MATLAB 7.01 [18] with machine accuracy equals $2.22 \times 10^{-16}$. We use $O b j_{\text {schur }}$ and $O b j_{\text {Newton }}$ respectively to denote the values of the departure from normality measure from the Schur and Schur-Newton algorithms (before and after the Newton refinement in Section 4).

Ex1. $n=4, m=2, \lambda_{\alpha}=\{1,1,1,1.0 e-8\}, \lambda_{\beta}=\{-20,-1,-3,-4\}$;

$$
\begin{gathered}
A=\left[\begin{array}{cccc}
-65 & 65 & -19.5 & 19.5 \\
0.1 & -0.1 & 0 & 0 \\
1 & 0 & -0.5 & -1 \\
0 & 0 & 0.4 & 0
\end{array}\right], E=\left[\begin{array}{cccc}
1000 & 0 & 0 & 0 \\
1 & 1 & 0 & 0 \\
1 & 90 & 0 & 1 \\
0 & 0 & 0 & 1
\end{array}\right], B=\left[\begin{array}{cc}
65 & 0 \\
0 & 0 \\
0 & 0 \\
0 & 0.4
\end{array}\right] ; \\
\text { Obj }_{\text {SCHUR }}=239, \text { Obj } j_{\text {Newton }}=14 ; \\
F=\left[\begin{array}{ccccc}
0.925 & -0.906 & 0.581 & -0.455 \\
11.075 & -12.4 & 17.8 & -7.25
\end{array}\right], G=\left[\begin{array}{cccc}
-15.3 & -0.005 & -0.860 & 0.440 \\
6.39 & 17.4 & -54.9 & 22.5
\end{array}\right] .
\end{gathered}
$$

Ex2. $n=4, m=2, \lambda_{\alpha}=\{1,1,1,1\}, \lambda_{\beta}=\{-1,-2,-3,-4\}$;

$$
\begin{gathered}
A=\left[\begin{array}{cccc}
0 & 0 & 0 & 0 \\
1 & 10 & 100 & 1000 \\
0 & 1 & 10 & 100 \\
0 & 0 & 1 & 10
\end{array}\right], E=\left[\begin{array}{cccc}
1 & 0 & 0 & 0 \\
1 & 0 & 0 & 0 \\
0 & 0 & 0 & 1 \\
0 & 0 & 1 & 1
\end{array}\right], \quad B=\left[\begin{array}{cc}
1 & 0 \\
0 & 1 \\
0 & 0 \\
0 & 0
\end{array}\right] ; \\
\text { Obj } j_{\text {SCHUR }}=30789, \quad \text { Obj jewton }=42 ; \\
F=\left[\begin{array}{cccc}
0.196 & -0.576 & -0.125 & -3.99 \\
-1.24 & -10.4 & -100 & -997
\end{array}\right], G=\left[\begin{array}{cccc}
-19.0 & -0.115 & 3.78 & -8.02 \\
17.8 & -0.036 & 4.73 & 1.40
\end{array}\right] .
\end{gathered}
$$


Ex3. $n=5, m=2, \lambda_{\alpha}=\{1,1,1,1,1\}, \lambda_{\beta}=\{-0.2,-0.5,-1,-1+i,-1-i\}$;

$$
\begin{aligned}
& A=\left[\begin{array}{ccccc}
-0.1094 & 0.0628 & 0 & 0 & 0 \\
1.306 & -2.132 & 0.9807 & 0 & 0 \\
0 & 1.595 & -3.149 & 1.547 & 0 \\
0 & 0.0355 & 2.632 & -4.257 & 1.855 \\
0 & 0.0023 & 0 & 0.1636 & -0.1625
\end{array}\right] \\
& E=\left[\begin{array}{lllll}
1 & 0 & 0 & 0 & 0 \\
1 & 0 & 0 & 0 & 0 \\
0 & 0 & 0 & 0 & 1 \\
0 & 0 & 1 & 0 & 1 \\
0 & 0 & 0 & 1 & 0
\end{array}\right], \quad B=\left[\begin{array}{cc}
0 & 0 \\
0.0638 & 0 \\
0.0838 & -0.1396 \\
0.1004 & -0.206 \\
0.0063 & -0.0128
\end{array}\right] \\
& \mathrm{Obj}_{\mathrm{SCHUR}}=60, \mathrm{Obj}_{\mathrm{Newton}}=54 ; \\
& F=\left[\begin{array}{lllll}
-46.4 & 50.9 & -55.0 & 6.12 & 29.5 \\
-23.3 & 22.1 & -25.1 & 5.53 & 21.4
\end{array}\right], G=\left[\begin{array}{lllll}
-22.5 & -2.46 & 17.5 & -216 & 21.2 \\
-18.6 & -2.05 & 21.0 & -150 & 21.8
\end{array}\right] \text {. }
\end{aligned}
$$

\section{Comments.}

(1) For Ex1 and Ex2 with real eigenvalues, the starting vectors from the Schur algorithm in Section 3 fall within the domain of convergence for the SchurNewton algorithm. This coincides with our experience with other RPAP_DS with real eigenvalues. The subsequent Newton refinement produces a local minimum which improves the robustness measure substantially.

(2) For the RPAP_DS with complex eigenvalues like Ex3, the starting vectors from Schur are often infeasible. Preliminary correction by Newton's iteration can be applied to the constraints in Optimization Problem 2, with gradient $J_{2}$. This produces a feasible starting vector for the Schur-Newton algorithm. However, the improvement in the robustness measure can be limited, as shown in Ex3. Apart from having an infeasible starting vector far from a local minimum, the main difficulty lies in the choice of finding accurate starting values for the Lagrange multipliers. However, improvements are still possible theoretically and achieved in practice, as seen in Ex3.

\section{ACKNOWLEDGMENT}

We would like to thank Professors Wen-Wei Lin (National Tsinghua University) and Shu-Fang $\mathrm{Xu}$ (Peking University) for various interesting discussions and much encouragement. 


\section{REFERENCES}

1. C. Beattie and I. C. F. Ipsen, Inclusion regions for matrix eigenvalues, Lin. Alg. Appl., 358 (2003), 281-291.

2. D. L. Chu, H. C. Chan and D. W. C. Ho, Regularization of singular systems by derivatives and proportional output feedback, SIAM J. Matrix Anal. Appl., 19 (1998), 1-38.

3. K.-W. E. Chu, A pole assignment algorithm for linear state feedback, Syst. Control Letts., 49 (1986), 289-299.

4. K.-W. E. Chu, On the controllability of descriptor systems, Int. J. Control., 46 (1987), 1761-1770.

5. K.-W. E. Chu, A canonical form and a state feedback pole assignment algorithm for descriptor systems, IEEE Trans. Automat. Control, AC-33 (1988), 1114-1125.

6. K.-W. E. Chu, Optimization and pole assignment in control system design, Int. J. Appl. Math. Comput. Sci., 11 (2001), 1035-1053.

7. K.-W. E. Chu, Pole assignment for second-ordered systems, Mech. Syst. Signal Processing, 16 (2002), 39-59.

8. K.-W. E. Chu, Pole assignment via the Schur form, Syst. Control Letts., 56 (2007), 303-314.

9. K.-W. E. Chu and B. N. Datta, Numerically robust pole assignment for second-order systems, Int. J. Control, 64 (1996), 1113-1127.

10. G.-R. Duan and R. J. Patton, Robust pole assignment in descriptor systems via proportional plus partial derivative state feedback, Int. J. Cont., 72 (1999), 1193-1203.

11. L. R. Flecther, Pole assignment and controllability subspaces in descriptor systems, Int. J. Control, 66 (1997), 677-709.

12. G. H. Golub and C. F. van Loan, Matrix Computations, 2nd Edn., Johns Hopkins University Press, Baltimore, MD, 1989.

13. L. Grippo and M. Sciandrone, Nonmonotone globalization techniques for the BarzilaiBorwein gradient method, Comput. Optim. Applics., 23 (2002), 143-169.

14. P. Henrici, Bounds for iterates, inverses, spectral variation and the field of values of nonnormal matrcies, Numer. Math., 4 (1962), 24-40.

15. J. Kautsky, N. K. Nichols and K.-W. E. Chu, Robust pole assignment in singular control systems, Lin. Alg. Appl., 121 (1989), 9-37.

16. J. Kautsky, N. K. Nichols and P. van Dooren, Robust pole assignment via in linear state feedback, Int. J. Control, 41 (1985), 1129-1155.

17. T. Li and K.-W. E. Chu, A Schur-Newton Algorithm for Robust Pole Assignment, Taiwanese J. Math., 11 (2007), 1485-1502.

18. Mathworks, MATLAB User's Guide, 2002. 
19. J.-G. Sun, Perturbation Theory of Matrices (in Chinese), 2nd ed., Science Press, 2001.

20. G. W. stewart and J.-G. Sun, Matrix Perturbation Theory, Academic Press, New York, 1990.

21. A. Varga, A Schur method for pole assignment, IEEE Trans. Autom. Control, AC-26 (1981), 517-519.

22. A. Varga, Computation of normalized coprime factorizations of rational matrices, Lin. Alg. Applic., 271 (1998), 83-115.

23. A. Varga, Robust pole assignment techniques for descriptor systems, Proc. Mathematical Theory of Networks and Systems MTNS2000, Perpignon, 2000.

24. A. Varga, A numerically reliable approach to robust pole assignment for descriptor systems, Future Generation Computer Syst., 19 (2003), 1221-1230.

Tiexiang $\mathrm{Li}$

Department of Mathematics,

Southeast University,

Nanjing 211189, Jiangsu,

P. R. China

E-mail: feco@sohu.com

Eric King-wah Chu

School of Mathematical Sciences,

Monash University, Building 28,

VIC 3800, Australia

E-mail: eric.chu@sci.monash.edu.au 